\title{
Aspectos bioéticos del aborto en Chile (1)
}

El debate sobre el aborto provocado no es indiferente para nadie. Tensiona y compromete de alguna manera a toda nuestra sociedad. No puede ser de otra manera, ya que en él participan valores o principios que no son banales y nos comprometen profundamente como individuos y como organización social. En una perspectiva más global, puede interesar como un elemento decidor del tipo de sociedad en la que queremos vivir, o para establecer políticas públicas de asignación de recursos sanitarios o de regulación poblacional; $y$ en un sentido más particular puede involucrar directamente a individuos en decisiones que afectarían radicalmente a otros y a ellos mismos. Ambas esferas son importantes; en la primera las consecuencias de las decisiones comprometen a un gran número de personas y al "carácter" de una sociedad. En el segundo nivel la afectación es personal y/o para los más cercanos o significativos.

En todos esos niveles estamos comprometidos los médicos obstetras. En efecto, actuamos en forma organizada y con una perspectiva más global cuando participamos en nuestra sociedad científica que busca el desarrollo de la especialidad, el perfeccionamiento de cada uno de sus miembros y el aporte social de nuestro conocimiento. Actuamos a la vez en forma personal en cada una de nuestras decisiones clínicas, cada vez que interactuamos con nuestros pacientes buscando su bienestar.

Parece entonces indispensable que como sociedad científica participemos en este debate, no sólo como interlocutores válidos por la experiencia práctica y conocimiento científico que poseemos, sino porque en definitiva somos nosotros los que atendemos a las pacientes que sufren las situaciones críticas que hacen pensar en la posibilidad de un aborto, y nosotros los que tendríamos que efectuar los procedimientos para conseguir esos objetivos. Esa decisión y ejecución nos involucra personalmente, ya que nada le sucede al que sólo opina, pero el que se decide a ejecutar u omitir una intervención, se convierte en un agente con responsabilidad ética, ya que es causa de algún resultado.

Como no podemos eludir el dilema, ni como so- ciedad científica ni como médicos clínicos, sugiero enfrentarlo en forma directa y clara. Propongo un esfuerzo racional para lograr precisar cuáles son las acciones que nos corresponden realizar imperativamente como médicos obstetras, cuales no nos corresponde realizar, y en cuáles tenemos incertidumbres.

Lo primero que tenemos que dilucidar es a quién debemos nosotros nuestros cuidados. Cuál es el objetivo, fin o meta que perseguimos como especialidad médica, o qué nos especifica como médicos, o dicho de otra forma, qué nos distingue de otros especialistas. La respuesta a estas interrogantes es clave, y nos permitirá precisar el campo de nuestra responsabilidad como obstetras o especialistas en medicina materno-fetal. Veamos, si somos médicos nos corresponde la salud de nuestros pacientes. La medicina desde tiempos inmemoriales se considera como aquella actividad que busca prevenir y curar las enfermedades de los pacientes. Ese es el objeto, fin o meta de la medicina, y frente a cada intento de otorgarle otros fines $u$ objetivos, se vuelve necesariamente a los principios y valores inherentes e inmanentes de la profesión (1).

Si a la cardiología como especialidad médica le corresponden las patologías del sistema cardiovascular, a la obstetricia le corresponden las patologías del embarazo, parto y puerperio y a la medicina materno-fetal, como subespecialidad de ella, le corresponde el tratamiento del feto in útero y aquellas patologías maternas en cuanto lo afectan. A cada subespecialidad o especialidad le corresponde un aspecto específico de la medicina, pero es obvio que todas ellas deben buscar el fin propio de ella, ya que de contrario dejarían de ser especialidades médicas. Todas ellas entonces buscan la salud de sus pacientes en sus campos más específicos.

Aparece entonces como contrario a la profesión médica el atentar contra la vida de un paciente. Cuesta mucho concebir que en una actividad alguien busque un resultado que vaya en contra del objetivo de ella. Así como sería incomprensible que un profesor busque que un alumno no aprenda, o que un piloto intente no llegar a su destino, también

\footnotetext{
${ }^{1}$ The Hastings Center Report (nov-dic, 1996), centro importante de Bioética, reunió a representantes de trece países en 1996 para volver a discutir los fines de la Medicina. Finalmente el trabajo propuso mantener los fines tradicionales de ella: La prevención de la enfermedad y de las lesiones, junto con la promoción y mantenimiento de la salud. El alivio del dolor y del sufrimiento causado por la enfermedad y las dolencias. La asistencia y curación de los enfermos, además del cuidado de los que no pueden ser curados. Evitar la muerte prematura y velar por una muerte en paz.
} 
resulta difícil de comprender que un médico intente afectar la salud o la vida de un paciente a su cargo. Es muy cierto que intervenciones médicas pueden tener como resultado un daño e incluso la muerte de un paciente, pero son efectos no deseados, inevitables o por último debidos a un actuar negligente, pero esos efectos nunca están en la intención del médico.

Si nos corresponde entonces como obstetras que una mujer y su hijo terminen su proceso de embarazo, parto y puerperio en las mejores condiciones de salud que nuestro estado del arte nos permite, si también nos corresponde como especialistas en medicina materno-fetal el tratamiento de las patologías fetales, o de aquellas afecciones maternas en cuanto afectan el desarrollo de su hijo in útero, es que no podemos permitirnos, y tendríamos que rechazar como contrarios a los principios de nuestra especialidad y de nuestra profesión, cualquier intervención que busque intencionalmente atentar contra la integridad de ese ser humano en gestación (2).

Se nos pueden hacer varias objeciones. La primera y más relevante es sobre el estatuto de paciente del embrión o feto. Claro, la conclusión "El médico respeta la vida del feto", derivada de las premisas "El médico respeta la vida de todo paciente y el feto es un paciente", se invalida si la segunda premisa del silogismo es falsa. Si no hay paciente, no hay obligación ética de respetar su integridad ni buscar su salud. No pretendo en estas líneas desarrollar razonamientos de orden científico, filosófico o religioso para justificar que sí es un paciente. Solamente me referiré a situaciones clínicas de las cuales se puede inferir, con una mínima lógica, que en el embrión o feto tenemos a un paciente.

Cuando tratamos a una mujer con amenaza de aborto, ¿estamos tratando a una paciente con una metrorragia por una de las tantas causas de ella? Al instalar un balón intratraqueal a un feto con hernia diafragmática, ¿estamos instalando ese balón en un cierto tipo extraño de tejido? Es evidente que nuestra intención al indicar reposo a esa mujer en el primer caso no es sólo inhibir el sangrado sino que alguien más sobreviva, y en el segundo caso, es también evidente que se decidió hacer un tratamiento a un paciente en el útero, ya que tiene mejor perspectiva de éxito que hacerlo una vez nacido. En ambos casos entonces, ni la mujer-paciente ni el médico se cuestionan sobre si el embrión o feto tiene la calidad de paciente. La conducta normal, habitual, racional, lógica y acorde a los fines de la medicina, es tratarlo entonces como tal.

La segunda objeción o dificultad se dirige hacia una consideración del feto o embrión como de paciente, pero de una categoría menor. Frases como "En nuestro servicio privilegiamos a la madre" o "Lo haremos porque ella es la única que tiene mayores posibilidades", indican que aunque sea cierto que en algunas situaciones clínicas que vivimos, las posibilidades de sobrevida de un embrión o feto sean bastante menores que las de la madre, se empieza a relativizar su estatuto de paciente y se tiende a ofrecerle menos cuidados o simplemente se atenta contra su vida. Todos hemos vivido por ejemplo, la situación -por suerte ya casi desterrada- de "aborto inevitable" en el caso de rotura ovular, que implicaba el vaciamiento del útero. También se puede ver esta actitud en casos de patologías fetales con pronóstico ominoso. Se puede entender que existan situaciones clínicas difíciles, en las cuales el obstetra se mueve en un equilibrio muy inestable con graves riesgos para ambos pacientes bajo su responsabilidad, pero difícilmente se puede considerar como equitativo y aceptable que se abandone a su suerte a uno de ellos, o peor aún, se atente contra su vida ante una situación difícil. De hecho, eso es lo propio de la medicina, actuar con diligencia en lo fácil y también en lo complicado.

Si bajo esta perspectiva, que es la perspectiva de la Medicina desde los tiempos de Hipócrates, analizamos las situaciones clínicas, actualmente en el debate público y en la consideración del Congreso Nacional, podremos aportar lo siguiente: en aquellas situaciones en que el médico tratante, después de hacer todos sus esfuerzos, llega a la convicción razonable que si continúa la gestación se morirá la madre, su obligación ética es interrumpir ese embarazo. De hecho así lo consideramos universalmente los obstetras. Ahora bien, en vista de precisar la razón de esa legitimidad, tenemos que indicar que esa acción es lícita y obligatoria no porque se está "privilegiando a la madre" o debido a que "es la única que se puede salvar". Es lícita y éticamente obligatoria para el tratante, debido a que es la única opción terapéutica también para el feto, ya que si se muere la madre inevitablemente también perecerá. En caso de ocurrir la muerte de él al nacer, sería una muerte lamentada por todos.

Distinta evaluación tendríamos que hacer en otras situaciones: en aquellas gestaciones producto de una violación y los embarazos con fetos con alguna condición de pronóstico ominoso. Si nos fijamos en lo que se busca con esas interrupciones, es que el feto muera. En el primer caso como intento de solución o medida paliativa para una paciente agredida brutalmente, y en el segundo para aliviar a una madre de una situación angustiosa. Debemos recordar aquí que tenemos bajo nuestro cuidado a dos pacientes, y nuestra responsabilidad es que ambos terminen el proceso de embarazo de la mejor forma posible. Nos corresponde poner al servicio de nuestros dos pacientes todos los recursos razonables, toda nuestra ciencia y experiencia para velar por la salud de ambos y que vivan lo que naturalmente les corresponde.

\footnotetext{
2 "El respeto de la vida humana desde su inicio y hasta su término constituye el fundamento básico del ejercicio profesional médico. Toda intervención médica realizada durante los nueve meses de gestación, deberá velar siempre por el mejor interés de la madre y del hijo." Art. 8. Código de Ética. Colegio Médico de Chile A.G.
} 
Como médicos especialistas en obstetricia o en medicina materno fetal debemos actuar siempre buscando la salud de nuestros pacientes. Es cierto que nos enfrentamos muchas veces con problemas clínicos difíciles y decisiones que hay que tomar rápidamente. Frecuentemente no sabemos con claridad cuál es la mejor acción. Es más fácil ver en esos momentos lo que es mejor para la madre, y se nos oscurece el bien para su hijo. En esos precisos casos, preguntémonos en la intimidad de nuestra deliberación si estamos haciendo nuestro mejor esfuerzo para nuestros dos pacientes. Si la respuesta es positiva seguramente estaremos ejer- ciendo la medicina y nuestra especialidad como se ha entendido desde el inicio de la profesión. A la Sociedad Chilena de Obstetricia y Ginecología, al ser la sociedad que nos congrega como especialistas, le corresponde, que sus asociados se formen bajo los mejores estándares clínicos y éticos, y también orientar a nuestros conciudadanos sobre los valores que rigen nuestra profesión y especialidad.

Dr. Mauricio Besio Rollero. División de Obstetricia y Ginecología. Centro de Bioética. Pontificia Universidad Católica de Chile. 